\title{
A pexider difference for a pexider functional equation
}

\author{
Abbas Najati ${ }^{*}$, Saeid Ostadbashi ${ }^{2}$, Gwang Hui $\mathrm{Kim}^{3}$ and Sooran Mahmoudfakhe ${ }^{2}$
}

\footnotetext{
* Correspondence: a.nejati@yahoo. com

${ }^{1}$ Department of Mathematical Sciences, University of Mohaghegh Ardabili, Ardabil 56199-11367, Iran Full list of author information is available at the end of the article
}

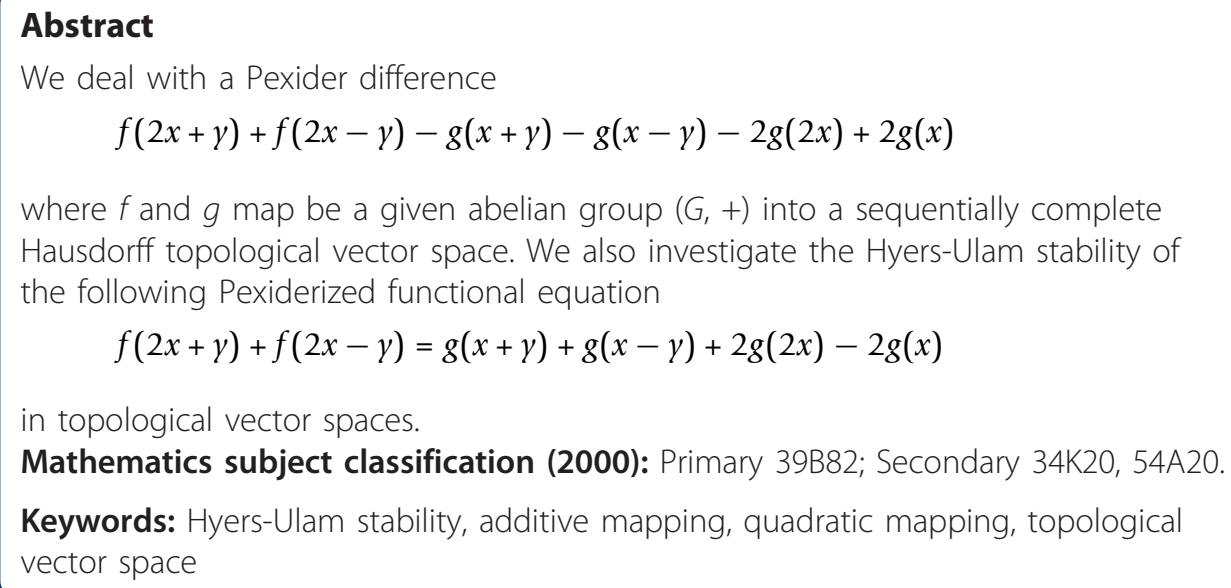

$$
f(2 x+y)+f(2 x-y)=g(x+y)+g(x-y)+2 g(2 x)-2 g(x)
$$

in topological vector spaces.

Mathematics subject classification (2000): Primary 39B82; Secondary 34K20, 54A20.

Keywords: Hyers-Ulam stability, additive mapping, quadratic mapping, topological vector space

\section{Introduction and preliminaries}

In 1940, Ulam [1] proposed the general stability problem: Let $G_{1}$ be a group, $G_{2}$ be a metric group with the metric $d$. Given $\varepsilon>0$, does there exists $\delta>0$ such that if a function $h: G_{1} \rightarrow G_{2}$ satisfies the inequality

$$
d(h(x y)-h(x) h(y))<\delta, \quad\left(x, y \in G_{1}\right),
$$

then there is a homomorphism $H: G_{1} \rightarrow G_{2}$ with

$$
d(h(x), H(x))<\varepsilon, \quad\left(x \in G_{1}\right) ?
$$

Hyers [2] gave a partial affirmative answer to the question of Ulam in the context of Banach spaces. In 1950, Aoki [3] extended the theorem of Hyers by considering the unbounded cauchy difference inequality

$$
\|f(x+y)-f(x)-f(y)\| \leq \varepsilon\left(\|x\|^{p}+\|y\|^{p}\right) \quad(\varepsilon>0, p \varepsilon[0,1)) .
$$

In 1978, Rassias [4] also generalized the Hyers' theorem for linear mappings under the assumption $t \mapsto f(t x)$ is continuous in $t$ for each fixed $x$.

Recently, Adam and Czerwik [5] investigated the problem of the Hyers-Ulam stability of a generalized quadratic functional equation in linear topological spaces. Najati and Moghimi [6] investigated the Hyers-Ulam stability of the functional equation

(c) 2012 Najati et al; licensee Springer. This is an Open Access article distributed under the terms of the Creative Commons Attribution License (http://creativecommons.org/licenses/by/2.0), which permits unrestricted use, distribution, and reproduction in any medium, provided the original work is properly cited. 


$$
f(2 x+y)+f(2 x-y)=f(x+y)+f(x-y)+2 f(2 x)-2 f(x)
$$

in quasi-Banach spaces. In this article, we prove that the Pexiderized functional equation

$$
f(2 x+y)+f(2 x-y)=g(x+y)+g(x-y)+2 g(2 x)-2 g(x)
$$

is stable for functions $f$, $g$ defined on an abelian group and taking values in a topological vector space.

Throughout this article, let $G$ be an abelian group and $X$ be a sequentially complete Hausdorff topological vector space over the field $\mathbb{Q}$ of rational numbers.

A mapping $f: G \rightarrow X$ is said to be quadratic if and only if it satisfies the following functional equation

$$
f(x+y)+f(x-y)=2 f(x)+2 f(y)
$$

for all $x y \in G$. A mapping $f G \rightarrow X$ is said to be additive if and only if it satisfies $f$ $(x+y)=f(x)+f(y)$ for all $x y \varepsilon G$. For a given $f: G \rightarrow X$, we will use the following notation

$$
D f(x, y):=f(2 x+y)+f(2 x-y)-f(x+y)-f(x-y)-2 f(2 x)+2 f(x) .
$$

For given sets $A B \subseteq X$ and a number $k \in \mathbb{R}$, we define the well known operations

$$
A+B:=\{a+b: a \in A, b \in B\}, k A:=\{k a: a \in A\} .
$$

We denote the convex hull of a set $U \subseteq X$ by $\operatorname{conv}(U)$ and by $\bar{U}$ the sequential closure of $U$. Moreover it is well know that:

(1) If $A \subseteq X$ are bounded sets, then $\operatorname{conv}(A)$ and $\bar{A}$ are bounded subsets of $X$.

(2) If $A, B \subseteq X$ and $\alpha \beta \in \mathbb{R}$, then $\alpha \operatorname{conv}(A)+\beta \operatorname{conv}(B)=\operatorname{conv}(\alpha A+\beta B)$.

(3) Let $X_{1}$ and $X_{2}$ be linear spaces over $\mathbb{R}$. If $f: X_{1} \rightarrow X_{2}$ is a additive (quadratic) function, then $f(r x)=r f(x)\left(f(r x)=r^{2} f(x)\right)$, for all $x \in X_{1}$ and all $r \in \mathbb{Q}$.

\section{Main results}

We start with the following lemma.

Lemma 2.1. Let $G$ be a 2-divisible abelian group and $B \subseteq X$ be a nonempty set. If the functions $f, g: G \rightarrow X$ satisfy

$$
f(2 x+y)+f(2 x-y)-g(x+y)-g(x-y)-2 g(2 x)+2 g(x) \in B
$$

for all $x, y \in G$, then

$$
\begin{aligned}
& D f(x, y) \in 2 \operatorname{conv}(B-B), \\
& D g(x, y) \in \operatorname{conv}(B-B)
\end{aligned}
$$

for all $x, y \in G$.

Proof. Putting $y=0$ in (2.1), we get

$$
2 f(2 x)-2 g(2 x) \in B
$$


for all $x \in G$. If we replace $x$ by $\frac{1}{2} x$ in (2.4), then we have

$$
f(x)-g(x) \in \frac{1}{2} B
$$

for all $x \in G$. It follows from (2.5) and (2.1) that

$$
\begin{aligned}
D f(x, y)= & f(2 x+y)+f(2 x-y)-g(x+y)-g(x-y)-2 g(2 x)+2 g(x) \\
& -[f(x+y)-g(x+y)]-[f(x-y)-g(x-y)] \\
& -[2 f(2 x)-2 g(2 x)]+[2 f(x)-2 g(x)] \\
\in & 2 \operatorname{conv}(B-B) .
\end{aligned}
$$

Moreover, we have

$$
\begin{aligned}
D g(x, y)= & f(2 x+y)+f(2 x-y)-g(x+y)-g(x-y)-2 g(2 x)+2 g(x) \\
& -[f(2 x+y)-g(2 x+y)]-[f(2 x-y)-g(2 x-y)] \\
\in & \operatorname{conv}(B-B) .
\end{aligned}
$$

Theorem 2.2. Let $G$ be a 2-divisible abelian group and $B \subseteq X$ be a bounded set. Suppose that the odd functions $f, g: G \rightarrow X$ satisfy (2 1) for all $x, y \in G$. Then there exists exactly one additive function $\mathcal{A}: G \rightarrow X$ such that

$$
\mathcal{A}(x)-f(x) \in 4 \overline{\operatorname{conv}(B-B)}, \quad \mathcal{A}(x)-g(x) \in \overline{\operatorname{conv}(B-B)}
$$

for all $x \in G$. Moreover the function $\mathcal{A}$ is given by

$$
\mathcal{A}(x)=\lim _{n \rightarrow \infty} \frac{1}{2^{n}} f\left(2^{n} x\right)=\lim _{n \rightarrow \infty} \frac{1}{2^{n}} g\left(2^{n} x\right)
$$

for all $x \in G$. Moreover, the convergence of the sequences are uniform on $G$.

Proof. By Lemma 2.1, we get (2.2). Setting $y=x, y=3 x$ and $y=4 x$ in (2.2), we get

$$
\begin{aligned}
& f(3 x)-3 f(2 x)+3 f(x) \in 2 \operatorname{conv}(B-B), \\
& f(5 x)-f(4 x)-f(2 x)+f(x) \in 2 \operatorname{conv}(B-B), \\
& f(6 x)-f(5 x)+f(3 x)-3 f(2 x)+2 f(x) \in 2 \operatorname{conv}(B-B)
\end{aligned}
$$

for all $x \in G$. It follows from (2.7), (2.8), and (2.9) that

$$
f(6 x)-f(4 x)-f(2 x) \in 6 \operatorname{conv}(B-B)
$$

for all $x \in G$. So

$$
f(3 x)-f(2 x)-f(x) \in 6 \operatorname{conv}(B-B)
$$

for all $x \in$ G. Using (2.7) and (2.10), we obtain

$$
\frac{1}{2} f(2 x)-f(x) \in 2 \operatorname{conv}(B-B)
$$


for all $x \in G$. Therefore

$$
\begin{aligned}
\frac{1}{2^{n}} f\left(2^{n} x\right)-\frac{1}{2^{m}} f\left(2^{m} x\right) & =\sum_{k=m}^{n-1}\left[\frac{1}{2^{k+1}} f\left(2^{k+1} x\right)-\frac{1}{2^{k}} f\left(2^{k} x\right)\right] \\
& \in \sum_{k=m}^{n-1} \frac{2}{2^{k}} \operatorname{conv}(B-B) \\
& \subseteq \frac{4}{2^{m}} \operatorname{conv}(B-B)
\end{aligned}
$$

for all $x \in G$ and all integers $n>m \geq 0$. Since $B$ is bounded, we conclude that conv $(B-B)$ is bounded. It follows from $(2.11)$ and boundedness of the set $\operatorname{conv}(B-B)$ that the sequence $\left\{\frac{1}{2^{n}} f\left(2^{n} x\right)\right\}$ is (uniformly) Cauchy in $X$ for all $x \in G$. Since $X$ is a sequential complete topological vector space, the sequence $\left\{\frac{1}{2^{n}} f\left(2^{n} x\right)\right\}$ is convergent for all $x$ $\in G$, and the convergence is uniform on $G$. Define

$$
\mathcal{A}_{1}: G \rightarrow X, \quad \mathcal{A}_{1}(x):=\lim _{n \rightarrow \infty} \frac{1}{2^{n}} f\left(2^{n} x\right) .
$$

Since $\operatorname{conv}(B-B)$ is bounded, it follows from (2.2) that

$$
D \mathcal{A}_{1}(x, y)=\lim _{n \rightarrow \infty} \frac{1}{2^{n}} D f\left(2^{n} x, 2^{n} y\right)=0
$$

for all $x y \in G$. So $\mathcal{A}_{1}$ is additive (see [6]). Letting $m=0$ and $n \rightarrow \infty$ in (2.11), we get

$$
\mathcal{A}_{1}(x)-f(x) \in 4 \overline{\operatorname{conv}(B-B)}
$$

for all $x \in$ G. Similarly as before applying (2.3) we have an additive mapping $\mathcal{A}_{2}: G \rightarrow X$ defined by $\mathcal{A}_{2}(x):=\lim _{n \rightarrow \infty} \frac{1}{2^{n}} g\left(2^{n} x\right)$ which is satisfying

$$
\mathcal{A}_{2}(x)-g(x) \in 2 \overline{\operatorname{conv}(B-B)}
$$

for all $x \in G$. Since $B$ is bounded, it follows from (2.5) that $\mathcal{A}_{1}=\mathcal{A}_{2}$. Letting $\mathcal{A}:=\mathcal{A}_{1}$, we obtain (2.6) from (2.12) and (2.13).

To prove the uniqueness of $\mathcal{A}$, suppose that there exists another additive function $\mathcal{A}^{\prime}$ : $G \rightarrow X$ satisfying (2.6). So

$$
\mathcal{A}^{\prime}(x)-\mathcal{A}(x)=\left[\mathcal{A}^{\prime}(x)-f(x)\right]+[f(x)-\mathcal{A}(x)] \in 8 \overline{\operatorname{conv}(B-B)}
$$

for all $x \in G$. Since $\mathcal{A}^{\prime}$ and $\mathcal{A}$ are additive, replacing $x$ by $2^{n} x$ implies that

$$
\mathcal{A}^{\prime}(x)-\mathcal{A}(x) \in \frac{8}{2^{n}} \overline{\operatorname{conv}(B-B)}
$$

for all $x \in G$ and all integers $n$. Since $\overline{\operatorname{conv}(B-B)}$ is bounded, we infer $\mathcal{A}^{\prime}=\mathcal{A}$. This completes the proof of theorem.

Theorem 2.3 Let $G$ be a 2, 3-divisible abelian group and $B \subseteq X$ be a bounded set. Suppose that the even functions $f, g: G \rightarrow X$ satisfy (2 1) for all $x, y \in G$. Then there exists exactly one quadratic function $\mathcal{Q}: G \rightarrow$ Xsuch that

$$
\mathcal{Q}(x)-f(x)+f(0) \in 4 \overline{\operatorname{conv}(B-B)}, \mathcal{Q}(x)-g(x)+g(0) \in 2 \overline{\operatorname{conv}(B-B)}
$$


for all $x \in G$. Moreover, the function $\mathcal{Q}$ is given by

$$
\mathcal{Q}(x)=\lim _{n \rightarrow \infty} \frac{1}{4^{n}} f\left(2^{n} x\right)=\lim _{n \rightarrow \infty} \frac{1}{4^{n}} g\left(2^{n} x\right)
$$

for all $x \in G$. Moreover, the convergence of the sequences are uniform on $G$.

Proof. By replacing $y$ by $x+y$ in (2.2), we get

$$
\begin{aligned}
f(3 x+y)+f(x-y) & -f(2 x+y)-f(y) \\
& -2 f(2 x)+2 f(x) \in 2 \operatorname{conv}(B-B)
\end{aligned}
$$

for all $x, y \in G$. Replacing $y$ by $-y$ in (2.14), we get

$$
\begin{aligned}
f(3 x-y)+f(x+y) & -f(2 x-y)-f(y) \\
& -2 f(2 x)+2 f(x) \in 2 \operatorname{conv}(B-B)
\end{aligned}
$$

for all $x, y \in G$. It follows from (2.2), (2.14), and (2.15) that

$$
\begin{aligned}
f(3 x+y) & +f(3 x-y)-2 f(y) \\
& -6 f(2 x)+6 f(x) \in 6 \operatorname{conv}(B-B)
\end{aligned}
$$

for all $x, y \in G$. By letting $y=0$ and $y=3 x$ in (2.16), we get

$$
\begin{aligned}
& 2 f(3 x)-6 f(2 x)+6 f(x)-2 f(0) \in 6 \operatorname{conv}(B-B), \\
& f(6 x)-2 f(3 x)-6 f(2 x)+6 f(x)+f(0) \in 6 \operatorname{conv}(B-B)
\end{aligned}
$$

for all $x \in G$. Using (2.17) and (2.18), we obtain

$$
f(6 x)-4 f(3 x)+3 f(0) \in 12 \operatorname{conv}(B-B)
$$

for all $x \in G$. If we replace $x$ by $\frac{1}{3} x$ in (2.19), then

$$
f(2 x)-4 f(x)+3 f(0) \in 12 \operatorname{conv}(B-B)
$$

for all $x \in G$. Therefore

$$
\frac{1}{4^{n+1}} f\left(2^{n+1} x\right)-\frac{1}{4^{n}} f\left(2^{n} x\right)+\frac{3}{4^{n+1}} f(0) \in \frac{3}{4^{n}} \operatorname{conv}(B-B)
$$

for all $x \in G$ and all integers $n$. So

$$
\begin{aligned}
& \frac{1}{4^{n}} f\left(2^{n} x\right)-\frac{1}{4^{m}} f\left(2^{m} x\right) \\
& =\sum_{k=m}^{n-1} \frac{1}{4^{k+1}} f\left(2^{k+1} x\right)-\frac{1}{4^{k}} f\left(2^{k} x\right) \\
& \in-\sum_{k=m}^{n-1} \frac{3}{4^{k+1}} f(0)+\sum_{k=m}^{n-1} \frac{3}{4^{k}} \operatorname{conv}(B-B) \\
& \subseteq-\sum_{k=m}^{n-1} \frac{3}{4^{k+1}} f(0)+\frac{1}{4^{m-1}} \operatorname{conv}(B-B)
\end{aligned}
$$

for all $x \in G$ and all integers $n>m \geq 0$. It follows from (2.21) and boundedness of the set $\operatorname{conv}(B-B)$ that the sequence $\left\{\frac{1}{4^{n}} f\left(2^{n} x\right)\right\}$ is (uniformly) Cauchy in $X$ for all $x \in$ $G$. The rest of the proof is similar to proof of of Theorem 2.2. 
Remark 2.4. If the functions $f, g: G \rightarrow X$ satisfy (2.1), where $f$ is even (odd) and $g$ is odd (even), then it is easy to show that $f$ and $g$ are bounded.

Author details

${ }^{1}$ Department of Mathematical Sciences, University of Mohaghegh Ardabili, Ardabil 56199-11367, Iran ${ }^{2}$ Department of Mathematics, Urmia University, Urmia, Iran ${ }^{3}$ Department of Applied Mathematics, Kangnam University, Giheung-gu, Yongin, Gyoenggi 446-702, Republic of Korea

\section{Authors' contributions}

All authors carried out the proof. All authors conceived of the study, and participated in its design and coordination. All authors read and approved the final manuscript.

\section{Competing interests}

The authors declare that they have no competing interests.

Received: 27 June 2011 Accepted: 6 March 2012 Published: 6 March 2012

\section{References}

1. Ulam, SM: Problem in Modern Mathematics, Science edn. Wiley, New York (1960)

2. Hyers, DH: On the stability of the linear functional equation. Proc Nat Acad Sci USA. 27, 222-224 (1941). doi:10.1073/ pnas.27.4.222

3. Aoki, T: On the stability of linear trasformation in Banach spaces. J Math Soc Japan. 2, 64-66 (1950). doi:10.2969/jmsj/ 00210064

4. Rassias, ThM: On the stability of the linear mapping in Banach spaces. Proc Amer Math Soc. 72, 297-300 (1978). doi:10.1090/S0002-9939-1978-0507327-1

5. Adam, M, Czerwik, S: On the stability of the quadratic functional equation in topological spaces. Banach J Math Anal. 1 , 245-251 (2007)

6. Najati, A, Moghimi, MB: Stability of a functional equation deriving from quadratic and additive functions in quasiBanach spaces. J Math Anal Appl. 337, 399-415 (2008). doi:10.1016/j.jmaa.2007.03.104

doi:10.1186/1687-1847-2012-26

Cite this article as: Najati et al:: A pexider difference for a pexider functional equation. Advances in Difference Equations 2012 2012:26.

\section{Submit your manuscript to a SpringerOpen ${ }^{\circ}$} journal and benefit from:

- Convenient online submission

Rigorous peer review

- Immediate publication on acceptance

- Open access: articles freely available online

- High visibility within the field

- Retaining the copyright to your article

Submit your next manuscript at $>$ springeropen.com 